\section{A) Check for updates}

Cite this: Food Funct., 2020, 11, 9177

\title{
Effect of dietary EPA and DHA on murine blood and liver fatty acid profile and liver oxylipin pattern depending on high and low dietary n6-PUFA $\dagger$
}

\author{
Laura Kutzner, ${ }^{a}$ Carsten Esselun, (D) ${ }^{\mathrm{b}}$ Nicole Franke, ${ }^{\mathrm{b}}$ Kirsten Schoenfeld, ${ }^{\mathrm{a}}$ \\ Gunter P. Eckert (D) ${ }^{b}$ and Nils Helge Schebb (D) *a
}

\begin{abstract}
The intake of long-chain n3-polyunsaturated fatty acids (PUFA), which are associated with beneficial health effects, is low in the Western diet, while the portion of dietary n6-PUFA and hence the n6/n3PUFA ratio is high. Strategies to improve the n3-PUFA status are n3-PUFA supplementation and/or lowering n6-PUFA intake. In the present study, mice were fed with two different sunflower oil-based control diets rich in linoleic (n6-high) or oleic acid (n6-low), either with low n3-PUFA content $(\sim 0.02 \%)$ as control or with $\sim 0.6 \%$ eicosapentaenoic acid (EPA) or docosahexaenoic acid (DHA). The n6-low diet had only little or no effect on levels of arachidonic acid (ARA) and its free oxylipins in liver tissue. Supplementation with EPA or DHA lowered ARA levels with an effect size of n6-high < n6-low. Blood cell \%EPA + DHA reached $>8 \%$ and $>11 \%$ in $n 6$-high and $n 6$-low groups, respectively. Elevation of EPA levels and EPA derived oxylipins was most pronounced in n6-low groups in liver tissue, while levels of DHA and DHA derived oxylipins were generally unaffected by the background diet. While the n6-low diet alone had no effect on blood and liver tissue ARA levels or n3-PUFA status, a supplementation of EPA or DHA was more effective in combination with an n6-low diet. Thus, supplementation of long-chain n3-PUFA combined with a reduction of dietary n6-PUFA is the most effective way to improve the endogenous n3-PUFA status.
\end{abstract}

Received 4th June 2020,

Accepted 23rd September 2020

DOI: $10.1039 /$ dOfo01462a

rsc.li/food-function

\section{Introduction}

Polyunsaturated fatty acids (PUFA) of the n3- and n6-family are essential nutrients because humans and most mammals do not possess the $\Delta 12$ - and $\Delta 15$-desaturases. ${ }^{1}$ Therefore, FA linoleic acid (LA, C18:2n6) and alpha-linolenic acid (ALA, C18:3n3) that are contained in e.g. plant seeds and nuts must be supplied by the diet and can be elongated/desaturated to longchain PUFA of the n6- and n3-family, which are crucial as constituents of cell membranes and precursors for bioactive lipid mediators. ${ }^{2}$ Attention has been paid to the positive effects of long-chain n3-PUFA eicosapentaenoic acid (EPA, C20:5n3) and docosahexaenoic acid (DHA, C22:6n3) and their health implications, e.g. in cardiovascular disease ${ }^{3}$ and inflammatory processes, ${ }^{4}$ while effects exerted by ALA are usually less pro-

${ }^{a}$ Chair of Food Chemistry, Faculty of Mathematics and Natural Sciences, University of Wuppertal, Gaussstr. 20, 42119 Wuppertal, Germany.E-mail: nils@schebb-web.de ; Fax: +49202439 3037; Tel: +492024393457

${ }^{b}$ Institute of Nutritional Sciences, Justus-Liebig-University, Wilhelmstr. 20, 35392 Giessen, Germany

$\dagger$ Electronic supplementary information (ESI) available. See DOI: 10.1039/ dofo01462a nounced. ${ }^{5}$ At least a part of the beneficial health effects of EPA and DHA are mediated by their eicosanoids and other oxylipins or shifts in the overall oxylipin profile. These bioactive lipid mediators are formed within the arachidonic acid (ARA, C20:4n6) cascade via three major enzymatic pathways and autoxidation. PUFA are converted by cyclooxygenases (COX) to prostanoids, i.e. prostaglandins and thromboxanes, however, also hydro(pero)xy PUFA formation can be catalyzed by COX enzymes. Lipoxygenases (LOX) are dioxygenases that lead to the formation of regio- and stereo-specific hydro(pero)xy fatty acids depending on the LOX isoform as well as leukotrienes and other multiple hydroxylated PUFA derivatives. Cytochrome P450 monooxygenases (CYP) are involved in the formation of terminal and mid-chain hydroxy PUFA as well as cis-epoxy PUFA, which can be enzymatically hydrolyzed by the soluble epoxide hydrolases (sEH) to the corresponding vicinal dihydroxy PUFA. ${ }^{6,7}$ Moreover, oxylipins can be formed by autoxidative processes leading to the formation of a vast number of prostaglandin-like isoprostanes and isofuranes, hydro(pero)xy fatty acids as well as cis- and trans-epoxy PUFA. ${ }^{8,9}$

Due to low EPA + DHA intake and relatively inefficient conversion of ALA to EPA and DHA in humans on a Western diet, blood and tissue levels of EPA + DHA are low. ${ }^{5,10}$ At the same 
time, there is an increased ingestion of n6-PUFA, e.g. due to consumption of plant oils rich in LA such as corn, sunflower or soybean oil leading to a high n6/n3-PUFA ratio in industrialized countries. ${ }^{10}$ Although PUFA consumption is associated with beneficial effects such as lowering serum cholesterol, ${ }^{11}$ high levels of n6-PUFA might favor the incorporation of ARA into blood/tissue phospholipids resulting in a high capacity for the formation of ARA derived, predominantly pro-inflammatory lipid mediators. ${ }^{12,13}$ Similarly, supplementation with long-chain n3-PUFA EPA and DHA increases both the endogenous levels of these PUFA as well as their oxylipins in a doseresponse manner. ${ }^{14,15}$ Moreover, due to competition for enzymatic conversion, n3-PUFA interventions may reduce the formation of ARA and other n6-PUFA derived oxylipins. ${ }^{15}$ For example, in mice and rats dietary EPA and DHA induced a shift in the overall tissue oxylipin profile by decreasing ARA derived oxylipins and increasing n3-PUFA derived oxylipins. ${ }^{16,17}$ Several oxylipins synthesized from EPA or DHA exert less inflammatory activity compared to those from ARA, e.g. EPA derived $\mathrm{LTB}_{5}$ vs. ARA derived $\mathrm{LTB}_{4}{ }^{18}$ or are directly involved in the resolution of inflammation, e.g. EPA and DHA derived specialized pro-resolving mediators (SPMs). ${ }^{19}$

Balancing dietary n6- and n3-PUFA and thereby endogenous n6- and n3-PUFA levels as well as their oxylipins may have positive implications for human health, e.g. in the context of cardiovascular disease. ${ }^{10}$ Modulation of the dietary n6/n3PUFA ratio can be achieved by different LA/ALA ratios in order to increase the EPA + DHA status. ${ }^{20}$ However, elevation of longchain n3-PUFA levels is more effective, when EPA and/or DHA are supplemented directly ${ }^{21}$ and low intake of (competing) n6PUFA could augment n3-PUFA uptake in blood and tissue lipid pools. In fact, increased n3-PUFA levels were observed in human erythrocytes and plasma when dietary LA was low and further elevated when combined with n3-PUFA supplementation. ${ }^{22,23}$ A combination of EPA + DHA supplementation with saturated fat resulted in a similar decrease of ARA and a more pronounced increase of EPA erythrocyte levels compared to a combination with an n6-PUFA-rich diet. ${ }^{24}$ Instead of increasing the intake of saturated fatty acids (SFA), dietary n6-PUFA can also be replaced by monounsaturated fatty acids (MUFA), such as oleic acid (OA, C18:1n9), which is contained in large amounts in e.g. olive oil. For example, lower ARA phospholipid levels when comparing dietary n6-PUFArich diets with olive oil-rich diets have been observed in mice ${ }^{25}$ and rats. ${ }^{26}$ Hence, a combination of MUFA and n3-PUFA is a promising approach in order to diminish excessive n6-PUFA intake, to reach a more balanced n6/n3-PUFA ratio and thereby increasing n3-PUFA bioavailability.

In order to get a deeper and more mechanistic understanding in how a combination of dietary MUFA (OA) supplemented with EPA or DHA affects tissue levels of n3- and n6-PUFA as well as the free oxylipins derived from those FA, we fed mice two different sunflower oil-based background diets (rich in LA or rich in OA) and supplemented either EPA or DHA. This background diet was chosen in order to keep influences from dietary n3-PUFA (e.g. ALA) minimal, maintain tissue SFA rela- tively constant and accept little influence related to the fat origin and other ingredients (e.g. antioxidants and polyphenols from olive oil). However, results using animal models may not be (completely) transferable to humans. Mice differ from humans regarding their FA and oxylipin metabolism. For example, mice can elongate and desaturate ALA more efficiently to EPA and DHA than humans. ${ }^{27}$ Thus, in the present study we used diets, which were low in ALA in order to assign observed effects to EPA/DHA administration rather than an efficient elongation/desaturation of ALA. It should be noted that this leads to a very low n3-PUFA content in the control groups. Analysis of a comprehensive set of both FA and free oxylipins allows to draw profound conclusions on how a background diet rich in LA or OA affects FA metabolism, how the tissue FA and oxylipin pattern can be further modified by a moderate dose of EPA or DHA and if the lowering n6-PUFA intake, n3-PUFA supplementation or a combination of both is most effective in improving the blood and tissue n3-PUFA status.

\section{Material and methods}

\subsection{Chemicals}

HPLC-grade methanol $(\mathrm{MeOH})$ and methyl tert-butyl ether (MTBE, Acros Organics) as well as LC-MS grade $\mathrm{MeOH}$, acetonitrile (ACN) and acetic acid (HOAc) were purchased from Fisher Scientific (Schwerte, Germany). Ammonium acetate was purchased from Merck (Darmstadt, Germany), potassium carbonate, ethyl acetate and acetyl chloride were obtained from Sigma Aldrich (Schnelldorf, Germany). Disodium hydrogen phosphate and $n$-hexane were obtained from Carl Roth (Karlsruhe, Germany). Oxylipin standard substances and deuterated internal oxylipin standards were purchased from Cayman Chemicals via local distributor Biomol (Hamburg, Germany), E-series resolvins RvE2, 18(S)-RvE3 and 18(R)-RvE3 were a kind gift of the lab of Makoto Arita (RIKEN Center for Integrative Medical Sciences, Japan), methyl pentacosanoate (FAME C25:0) was obtained from Santa Cruz Biotechnology (Heidelberg, Germany). PCR primers were purchased from Cayman Chemicals via local distributor Biomol (Hamburg, Germany).

\subsection{Animal experiment}

Three weeks old female NMRI (Navar Medical Research Institute) mice were purchased from Charles River (Sulzbach, Germany). For this study we only used female mice, because male mice would probably attack each other after being placed in different cages one after the other following randomization into the groups. Therefore, part of the variability regarding lipid metabolism could be based on the estrous cycle, which is generally 4-5 days in length. As experimentation was conducted in a continuous period, results should encompass all estrous states and not be biased by a specific state. Furthermore, studies have shown that phenotypic differences between female and male mice are rather small. ${ }^{28}$ Animals 
were housed according to the German guidelines for animal care and had, at all times, access to water and food ad libitum. Mice were maintained on a $12 \mathrm{~h}$ light/dark cycle. Mice were randomized into 6 groups with 8 mice each: control (c)/n6high, EPA/n6-high, DHA/n6-high, c/n6-low, EPA/n6-low and DHA/n6-low. Feeding started after one week of acclimatization, when mice were 4 weeks old. Animals were fed a total of 28 days, left-over feed was collected and weighed and new feed was distributed on a daily basis during the first week and biweekly during the following 3 weeks. Mice received a standard diet (product number: E15051, ssniff Spezialitäten $\mathrm{GmbH}$, Soest, Germany) with $10.1 \%$ total fat consisting of different oil mixtures: for n6-PUFA-high feed commercial refined sunflower oil (Thomy, Nestlé, Frankfurt, Germany) rich in LA was used and for n6-PUFA-low feed refined sunflower oil (Henry Lamotte Oils GmbH, Bremen, Germany) rich in OA was used. For EPA/n6-high and EPA/n6-low feed EPA was added in form of EPA-ethyl ester (EE) to LA-rich and OA-rich sunflower oil, respectively, resulting in a final EPA content of $5.5-5.7 \%$ of total FA (0.55-0.57\% in feed). For DHA/n6-high and DHA/n6low feed DHA was added in form of DHA-EE to LA-rich and OA-rich sunflower oil, respectively, resulting in a final DHA content of $5.4-5.7 \%$ of total FA $(0.54-0.57 \%$ in feed). All six oil mixtures contained $0.2 \%$ mixed tocopherol concentrate as antioxidant. The full composition of the diets and the FA profile of oils used as dietary fat are summarized in the ESI (Table S1†). Following the feeding period, mice were killed by cervical dislocation and decapitation. Whole blood was collected in EDTA tubes and centrifuged (10 min, $1500 g, 4{ }^{\circ} \mathrm{C}$ ). Plasma was collected, blood cells were resuspended in the respective volume of PBS buffer and stored at $-80{ }^{\circ} \mathrm{C}$ until analysis. Liver and other organs were quickly removed and frozen in liquid nitrogen before they were stored for longer periods of time at $-80^{\circ} \mathrm{C}$.

All experiments were carried out by individuals with appropriate training and experience in compliance with the requirements of the Federation of European Laboratory Animal Science Associations and the European Communities Council Directive (Directive 2010/63/EU). Experiments followed the institutional guidelines and were approved by the regional authority (Regierungspraesidium Darmstadt, Germany; \#V54 19 c 20/15 - FU8/22).

\subsection{Fatty acid analysis, GC-FID}

The method for FA analysis based on gas chromatography with flame ionization detection (GC-FID) as described ${ }^{29,30}$ was slightly modified. In brief, $20 \mu \mathrm{L}$ plasma (diluted with $30 \mu \mathrm{L}$ $\mathrm{H}_{2} \mathrm{O}$ ) or $100 \mu \mathrm{L}$ resuspended blood cells (BC) were mixed with $10 \mu \mathrm{L}$ methyl pentacosanoate (FAME C25:0, $750 \mu \mathrm{M}$ ) as internal standard (IS) as well as $10 \mu \mathrm{L} 0.02 \mathrm{mg} \mathrm{mL}^{-1} \mathrm{BHT}$ and EDTA in $\mathrm{MeOH} / \mathrm{H}_{2} \mathrm{O}(50 / 50, \mathrm{v} / \mathrm{v})$ as antioxidant and extracted with MTBE/MeOH. To $15 \pm 2 \mathrm{mg}$ liver tissue $10 \mu \mathrm{L}$ IS, $50 \mu \mathrm{L}$ $\mathrm{H}_{2} \mathrm{O}$ and $300 \mu \mathrm{L} \mathrm{MeOH}$ were added and tissue was homogenized in a ball mill using pre-cooled sample holders $25 \mathrm{~Hz}$, $5 \mathrm{~min}$ ) before MTBE/MeOH extraction. Dry lipid extracts were trans-esterified with methanolic hydrogen (90-95 $\left.{ }^{\circ} \mathrm{C}, 60 \mathrm{~min}\right)$ and the resulting FAMEs were injected into the GC system. Quantification was based on theoretical response factors (tRF) utilizing C25:0 as IS and FA profile was determined by relative peak areas (taking into account the tRF). The method covers a total of 39 FAMEs (excluding C25:0).

\subsection{Gene expression analysis by quantitative real-time PCR (qPCR)}

RNA was isolated using the RNAeasy Mini Kit (Qiagen, Hilden, Germany) following the manufacturer's instructions. Previously frozen liver tissue stored at $-80^{\circ} \mathrm{C}$, was thawed carefully before isolation. Following isolation, RNA concentration was determined via NanoDrop ${ }^{\mathrm{TM}}$ One/Onec (Thermo Fisher Scientific, Waltham, MA, USA) measuring the absorbance at 260 and $280 \mathrm{~nm}$. Ratios of absorbance 260/280 $\mathrm{nm}$ and 260/ $230 \mathrm{~nm}$ were used as a marker for RNA purity. To increase purity and remove residual genomic DNA, samples were treated with TURBO DNA-free ${ }^{\mathrm{TM}}$ Kit according to the manufacturer's instructions (Thermo Fisher Scientific, Waltham, MA, USA). Following purification, $1 \mu \mathrm{g}$ of total RNA was used for complementary cDNA synthesis using the iscript cDNA Synthesis Kit (BioRad, Munich, Germany) according to the manufacturer's instructions and BioRad Thermo Cycler T100 (BioRad, Munich, Germany). Until qPCR experiments, samples were stored at $-80{ }^{\circ} \mathrm{C}$. Quantitative real-time PCR was conducted using a CFX Connect ${ }^{\mathrm{TM}}$ system (BioRad, Munich, Germany). A list of all used oligonucleotide primer sequences, primer concentrations, product sizes and the applied PCR protocol can be found in the ESI (Table S5†). For all experiments, the initial denaturation step was conducted at $95{ }^{\circ} \mathrm{C}$ for $3 \mathrm{~min}$, followed by a varying number of cycles each consisting of a short denaturation phase $\left(95^{\circ} \mathrm{C}, 10 \mathrm{~s}\right)$, an annealing phase with differing temperatures $\left(53{ }^{\circ} \mathrm{C}\right.$ to $62.8^{\circ} \mathrm{C}$ ) and durations (30 s to $45 \mathrm{~s}$ ) depending on the primer used and a final elongation step $\left(72{ }^{\circ} \mathrm{C}, 30 \mathrm{~s}\right)$. Gene expression was analyzed with BioRad CFX Manager 3.1 software using the $2 \Delta \Delta$ Cq method. Results were normalized to expression levels of housekeeping genes beta-2-microglobuline (B2M) and phosphoglycerate kinase 1 (PGK1) and expressed in \% relative to the c/n6-high group.

\subsection{Oxylipin analysis, LC-MS/MS}

Oxylipin extraction and measurement was performed as described. ${ }^{31,32}$ In brief, to $50 \pm 5 \mathrm{mg}$ liver tissue $10 \mu \mathrm{L}$ antioxidant mixture, $10 \mu \mathrm{L}$ deuterated IS and $300 \mu \mathrm{L}$ ice-cold $\mathrm{MeOH}$ were added and tissue samples were homogenized using a ball mill as described above. After centrifugation of tissue homogenates (10 $\mathrm{min}, 20000 \mathrm{~g}, 4^{\circ} \mathrm{C}$ ), supernatants were diluted with $2.7 \mathrm{~mL} 0.1 \mathrm{M}$ disodium hydrogen phosphate buffer ( $\mathrm{pH} \mathrm{6)}$ and loaded onto the pre-conditioned solid phase extraction cartridges (SPE; Bond Elut Certify II, $200 \mathrm{mg}, 3 \mathrm{~mL}$; Agilent, Waldbronn, Germany). After washing the SPE cartridges with water and $\mathrm{MeOH} /$ water (50/50, v/v) and drying (30 $\mathrm{s},-200 \mathrm{mbar})$, oxylipins were eluted with ethyl acetate/ $n$-hexane $(75 / 25, \mathrm{v} / \mathrm{v})$ with $1 \%$ HOAc and evaporated to dryness in a vacuum concentrator. Reconstituted sample extracts were 
injected into the LC-MS/MS system (Agilent 1290 binary pump coupled to AB Sciex 6500 QTRAP MS) and separated on a Zorbax Eclipse Plus C18 reversed phase column (Agilent, Waldbronn, Germany) with a binary gradient using $0.1 \%$ HOAc with $5 \%$ solvent $\mathrm{B}$ as solvent $\mathrm{A}$ and $\mathrm{ACN} / \mathrm{MeOH} / \mathrm{HOAc}$ $(800 / 150 / 1, v / v / v)$ as solvent B. Measurement was carried out in scheduled multiple reaction monitoring (MRM) mode and quantification was carried out by external calibration (analyte/ IS area ratio). A total of 137 oxylipins was analyzed in the liver samples.

\subsection{Data analysis and statistical analysis}

For concentrations and relative levels of FA and oxylipins as well as relative gene expression mean values and standard error of the mean (SEM) were calculated using Microsoft Office Excel 2016 software (Redmond, WA, USA). Concentrations of FA and oxylipins were only quantified when exceeding the lower limit of quantification (LLOQ). If $\geq 50 \%$ of the concentrations within a group were $>$ LLOQ, concentrations <LLOQ were set to $\frac{1}{2}$ LLOQ and mean \pm SEM were calculated. If the mean did not exceed LLOQ or $<50 \%$ of the samples within a group were $>$ LLOQ, " $<$ LLOQ" is displayed for the whole group. For relative FA profile (\% of total FA) FA $<$ LLOQ were set to zero and mean \pm SEM were only calculated if $\geq 50 \%$ of the values within a group were $>$ LLOQ. Enzyme activity estimates were calculated based on product/precursor ratios as follows: D6D-index $=\mathrm{C} 18: 3 \mathrm{n} 6 / \mathrm{C} 18: 2 \mathrm{n} 6$, D5D-index $=\mathrm{C} 20: 4 \mathrm{n} 6 /$ $\mathrm{C} 20: 3 \mathrm{n} 6,{ }^{33}$ Elovl-index $=\mathrm{C} 22: 4 \mathrm{n} 6 / \mathrm{C} 20: 4 \mathrm{n} 6 .^{34}$ Calculation of $\%$ n3 in highly unsaturated fatty acids (HUFA) and \%n6 in HUFA was based on Lands et al. (2018). ${ }^{35}$ Statistical analysis was carried out using GraphPad Prism 6.01 software (GraphPad Software, San Diego, CA, USA). Statistically significant differences were determined by two-way ANOVA analysis (factor 1: background diet n6-high/n6-low; factor 2: n3-PUFA supplementation c/EPA/DHA) with Tukey's post-test for multiple comparisons (each mean with every other mean): ns $(p>0.05),{ }^{*}(p<$ $0.05),{ }^{* *}(p<0.01),{ }^{* * *}(p<0.001),{ }^{* * * *}(p<0.0001)$ and are summarized in the ESI (Table S8†). If a whole group was $<$ LLOQ, a modified two-way ANOVA (fewer groups) and/or a one-way ANOVA (comparison of groups with the same background diet) or a $t$-test was performed. For relative gene expression data outliers were removed based on ROUT outlier test $(Q=1 \%)$.

\section{Results}

\subsection{Animal food consumption and effect on body weight}

All experimental diets contained $10.1 \%$ fat and the total FA compositions of the oils used for the preparation of the feed were analyzed by GC-FID (ESI, Table S1†). Initial change of diets resulted in an increased consumption of food on the first day of the feeding period across all groups $v s$. food consumption for the rest of the feeding period $(34.0 \pm 0.9 \mathrm{~g} v s .26 .4 \pm$ $0.5 \mathrm{~g} ; p<0.0001)$. Neither the high/low n6-PUFA content of the diet nor the added EPA or DHA had an effect on food con- sumption (ESI, Table S2 $\dagger$ ). Weight of mice increased during the feeding period (ESI, Table S3†). Changes in weight were similar across all groups and independent from the different fats used in the diets.

\subsection{Relative fatty acid profile and n3-PUFA status}

Four weeks of feeding a diet with high or low content of n6PUFA and very low n3-PUFA content $(\sim 0.2 \%$ in feeding oils; ESI Table S1 $\dagger$ ) led to a strikingly different FA profile in murine liver tissue. This affected all FA classes (SFA, MUFA, PUFA) except \%n3-PUFA, which were similarly low $(2.6 \pm 0.3 \%$ (c/n6high) and $2.0 \pm 0.3 \%$ (c/n6-low); Fig. 1, ESI Table S4 $\dagger$ ). Noteworthy, lower \%SFA were attributable to higher total liver FA and accumulating OA in the c/n6-low compared to the c/n6high group. Consistent with the major dietary FA class (high LA vs. high OA), extreme differences were observed for \%MUFA and \%n6-PUFA: MUFA were the dominant FA class in the c/n6low group ( $59 \pm 3 \%$ ) and 2.0-fold higher compared to the c/n6high group $(29 \pm 3 \%)$, while n6-PUFA were the dominant FA class in the c/n6-high group $(37 \pm 2 \%)$ and 2.7 -fold higher compared to the c/n6-low group $(14 \pm 2 \%)$. Feeding the EPArich diet resulted in an increase of \%n3-PUFA in liver tissue regardless of the n6-PUFA content $(8.3 \pm 0.5 \%$ and $8.1 \pm 1.1 \%$ in $\mathrm{EPA} / \mathrm{n} 6$-high and $\mathrm{EPA} / \mathrm{n} 6$-low, respectively). A similar increase of \%n3-PUFA was observed in the DHA-supplemented groups $(9.6 \pm 0.8 \%$ and $9.9 \pm 0.7 \%$ in DHA/n6-high and DHA/ n6-low, respectively) (Fig. 1). In animals on an n6-PUFA-high diet neither EPA nor DHA supplementation significantly affected \%MUFA or \%n6-PUFA. In animals fed an n6-PUFA-low diet \%MUFA (c/n6-low $59 \pm 3 \%$ ) were decreased by supplementation with EPA to $48 \pm 3 \%$ and DHA to $45 \pm 2 \%$.

Regarding the n3-PUFA status, liver \%EPA + DHA was not different between the groups fed an n6-PUFA-high or n6-PUFAlow based diet (Fig. 1). In contrast, in BC, increase of \%EPA + DHA relative to the control group was in the order of EPA/n6high < EPA/n6-low < DHA/n6-high < DHA/n6-low (2.7- vs. 3.2fold increase for EPA/n6-high vs. EPA/n6-low; 3.3- vs. 3.8-fold increase for DHA/n6-high vs. DHA/n6-low). Thus, for both the n6-high and n6-low background diet DHA supplementation increased BC \%EPA + DHA more efficiently than EPA supplementation. Importantly, while \%EPA + DHA was not different between the control groups c/n6-high and c/n6-low, elevation of BC \%EPA + DHA upon n3-PUFA feeding was clearly more pronounced when combined with an n6-PUFAlow background diet. This was also the case in blood plasma. Levels of \%n3 in HUFA were consistently higher in the n6-low compared to n6-high-based groups in liver, $\mathrm{BC}$ and plasma and more effectively raised by DHA feeding in liver and BC. \%n6 in HUFA showed the reversed trend (Fig. 1). Complete relative FA profile in liver, $\mathrm{BC}$ and plasma are summarized in the ESI (Fig. S1 and S2 and Table S4 $\dagger$ ).

\subsection{Fatty acid concentrations in liver tissue}

Dominant FA in liver tissue was OA in both control groups, however, 3.3-fold lower in the c/n6-high compared to c/n6-low group $\left(16 \pm 4 \mathrm{~g} \mathrm{~kg}^{-1}\right.$ vs. $\left.53 \pm 10 \mathrm{~g} \mathrm{~kg}^{-1}\right)$. Higher dietary LA 

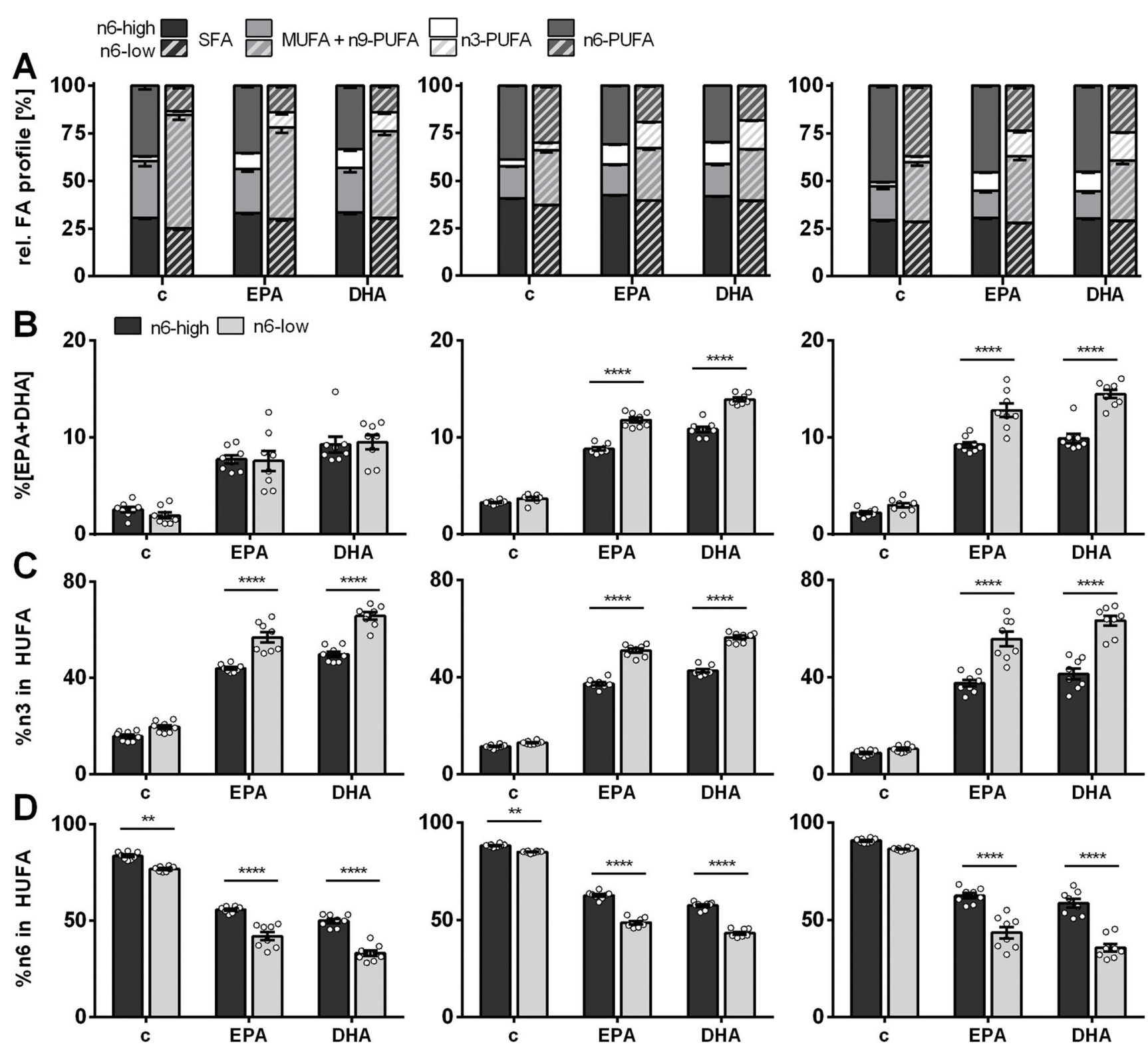

Fig. 1 Relative FA profile in mouse liver, blood cells and blood plasma. (A) Relative fatty acid profile of saturated (SFA), monounsaturated (MUFA), n3- and n6-polyunsaturated fatty acids (PUFA) as well as (B) \%EPA + DHA, (C) \%n3 in highly unsaturated fatty acids (HUFA) and (D) \%n6 in HUFA in liver tissue, blood cells and blood plasma of NMRI mice after 28 days of feeding an n6-PUFA-rich diet (dark grey) or an n6-PUFA-low diet (light grey) without (c) or with n3-PUFA supplementation (EPA, DHA). Sum of MUFA includes one n9-PUFA (C20:3n9). Shown are mean \pm SEM as well as individual values for (B-D) $(n=8)$. Statistically significant differences ( $p<0.05$; ${ }^{* *} p<0.01$; ${ }^{* * *} p<0.001$; $\left.{ }^{* * *} p<0.0001\right)$ were determined by two-way ANOVA with Tukey's post-test and are indicated for n6-high vs. n6-low groups (results for comparisons of all groups are summarized in the ESI, Table S8†).

resulted in 2.6-fold higher LA in the c/n6-high compared to c/n6-low group $\left(13.4 \pm 1.1 \mathrm{~g} \mathrm{~kg}^{-1}\right.$ vs. $\left.5.2 \pm 0.6 \mathrm{~g} \mathrm{~kg}^{-1}\right)$. Despite this pronounced difference in liver LA concentrations (Fig. 2), tissue ARA concentrations were not different between the two background diets $\left(6.5 \pm 0.2 \mathrm{~g} \mathrm{~kg}^{-1}\right.$ vs. $\left.5.6 \pm 0.3 \mathrm{~g} \mathrm{~kg}^{-1}\right)$. In contrast, downstream elongation/desaturation products adrenic acid (AdA, C22:4n6) and n6-docosapentaenoic acid (n6-DPA, C22:5n6) were lower in the c/n6-low group. Long-chain n3-
PUFA EPA, n3-DPA and DHA were similar in both control groups.

Upon n3-PUFA feeding, ARA concentrations were reduced in all feeding groups, while dihomo-gamma linolenic acid (DGLA, C20:3n6) levels were elevated in n6-high groups and LA levels were unaffected. Notably, ARA concentrations were lower in EPA/n6-low and DHA/n6-low groups compared to EPA/n6high and DHA/n6-high groups (Fig. 2). EPA, which did not 

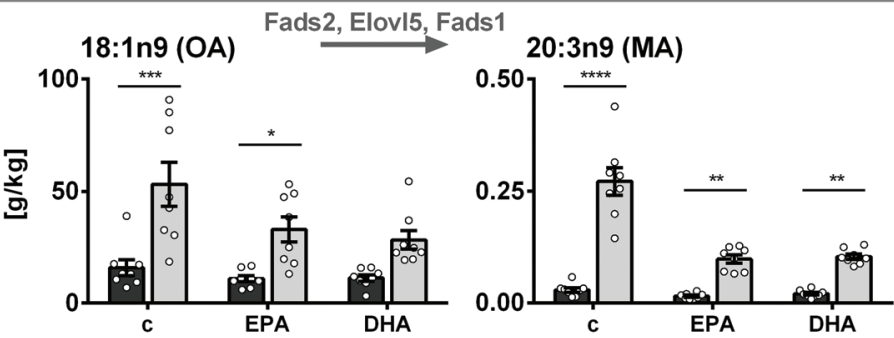

n6- and n3-PUFA
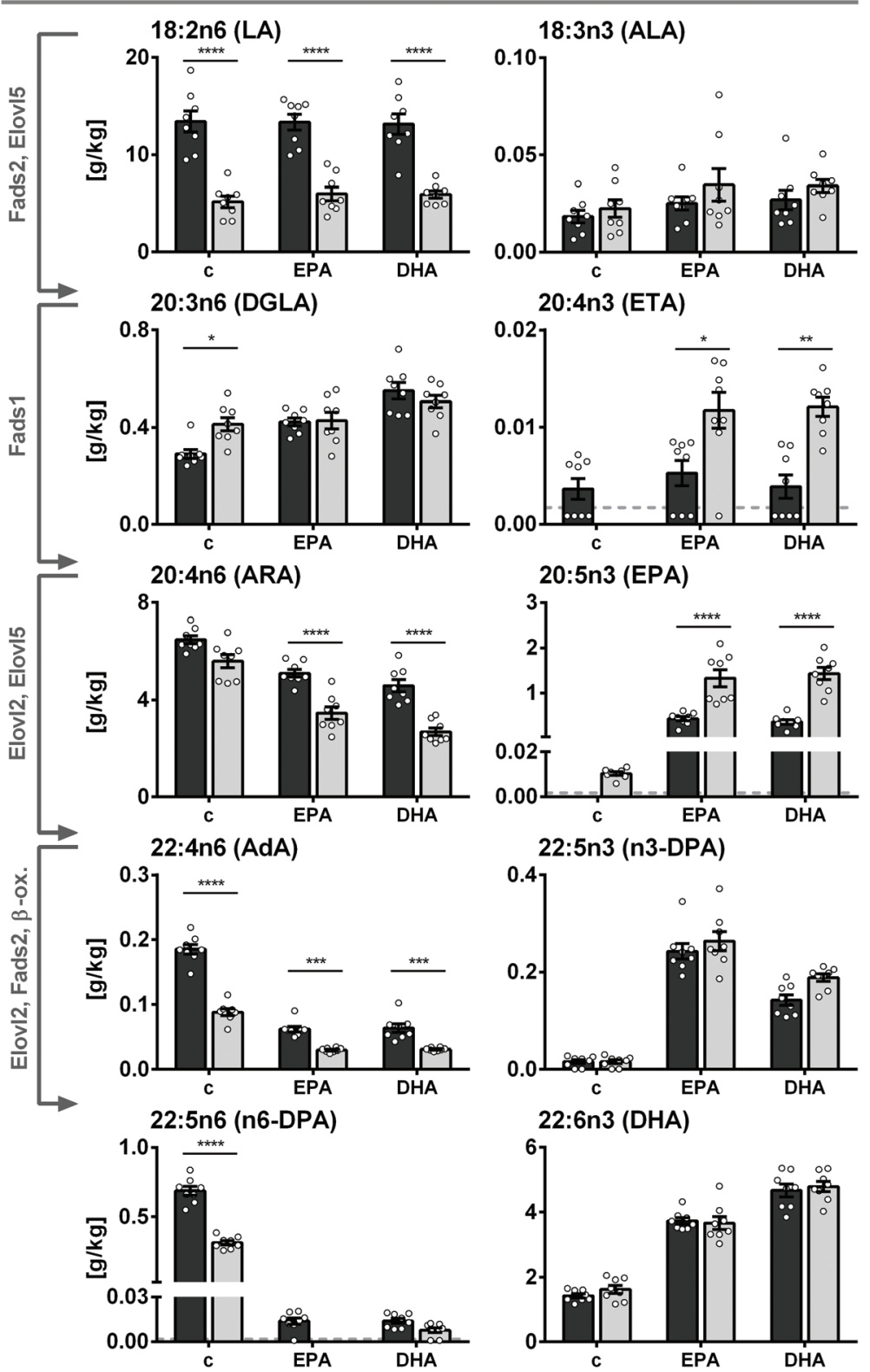

Fig. 2 FA concentrations in mouse liver tissue. Fatty acid concentrations [ $\mathrm{g} \mathrm{kg}^{-1}$ wet tissue] of n9-MUFA and -PUFA as well as n3- and n6-PUFA in liver tissue of NMRI mice after 28 days of feeding an n6-PUFA-rich diet (dark grey) or an n6-PUFA-low diet (light grey) without (c) or with n3-PUFA supplementation (EPA, DHA). Shown are mean \pm SEM as well as individual values $(n=8)$. If $>50 \%$ of the samples within one group were $<$ LLOQ (lower limit of quantification), no mean was calculated and the LLOQ is indicated as dotted line. Statistically significant differences $\left({ }^{*} p<0.05\right.$; ${ }^{* *} p<$

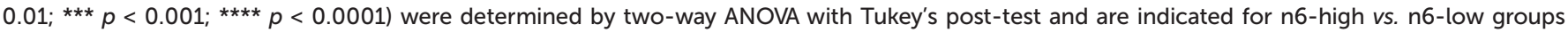
(results for comparisons of all groups are summarized in the ESI, Table S8 + ). Arrows and gene names indicate enzymes involved in FA desaturation and elongation and proposed formation routes adapted from Guillou et al. (2010). ${ }^{50}$ 
exceed the LLOQ in c/n6-high, was increased to $0.43 \pm 0.05 \mathrm{~g}$ $\mathrm{kg}^{-1}$ in the EPA/n6-high group. In the EPA/n6-low group, EPA increased from $0.010 \pm 0.001 \mathrm{~g} \mathrm{~kg}^{-1}$ (c/n6-low) to $1.3 \pm 0.2 \mathrm{~g}$ $\mathrm{kg}^{-1}$ corresponding to a 130-fold increase and 3.1-fold higher concentration compared to the n6-PUFA-high based diet. Interestingly, DHA supplementation had a similar effect on EPA concentrations in liver tissue (DHA/n6-high: $0.36 \pm 0.05 \mathrm{~g}$ $\mathrm{kg}^{-1}$, DHA/n6-low: $1.4 \pm 0.1 \mathrm{~g} \mathrm{~kg}^{-1}$ ). The elongation product of EPA, n3-DPA, was effectively elevated by both EPA and DHA supplementation, however, more pronounced in EPA-fed groups. The n6-PUFA background diet had no effect on n3DPA levels (Fig. 2). Liver tissue DHA concentrations increased upon EPA feeding and were more efficiently elevated in DHAfed groups. In contrast to EPA levels, DHA concentrations did not differ between the groups fed the n6-high or n6-low background diets (Fig. 2). All liver FA concentrations and statistical analyses are summarized in the ESI (Tables S4A-1 and S8A-1†).

\subsection{Fatty acid metabolizing enzyme expression in liver}

Gene expression of several markers of $\beta$-oxidation as well as FA elongation and desaturation were assessed via transcription analysis in qPCR experiments (ESI, Tables S5 and 6†). Both n3-
PUFA supplementation and the n6-low/n6-high background diet had only little or no effect on the gene expression of the key players of $\beta$-oxidation (ESI, Fig. S3 $\dagger$ ). Hadha and Hadhb, which are involved in the mitochondrial $\beta$-oxidation were statistically not different among all groups. This was also the case for the peroxisomal equivalents Ehhadh and Hsd17b4 as well as carnitine palmitoyltransferase $1 \mathrm{a}$ and 2 (Cpt1a/Cpt2). Enzymes which act as "auxiliary enzymes" in $\beta$-oxidation of long-chain PUFA, i.e. 2,4-dienoyl-CoA reductase 1 (Decr1) and 2 (Decr2) as well as cis- $\Delta^{3}$-enoyl-CoA isomerase 1 (Eci1) and 2 (Eci2), were unaffected by the n6-high and n6-low background diets (Fig. 3A). However, Eci1 and Eci2 mRNA levels in DHA/ n6-low compared to the c/n6-low group were increased.

Enzymes involved in FA desaturation and elongation, i.e. $\Delta 5$-desaturase (D5D; Fads1), $\Delta 6$-desaturase (D6D; Fads2), elongases 2 (Elovl2) and 5 (Elovl5), were not significantly affected by the diet (Fig. 3B). Estimates for enzyme activity based on FA product/precursor ratios were consistently lower for n3-PUFA supplementation (D5D-, D6D-, Elovl-index). The n6-PUFA background diet affected the D5D- and Elovl-indices, which were always lower in the n6-low groups, except for the Elovl-index in DHA-fed groups (Fig. 3C).
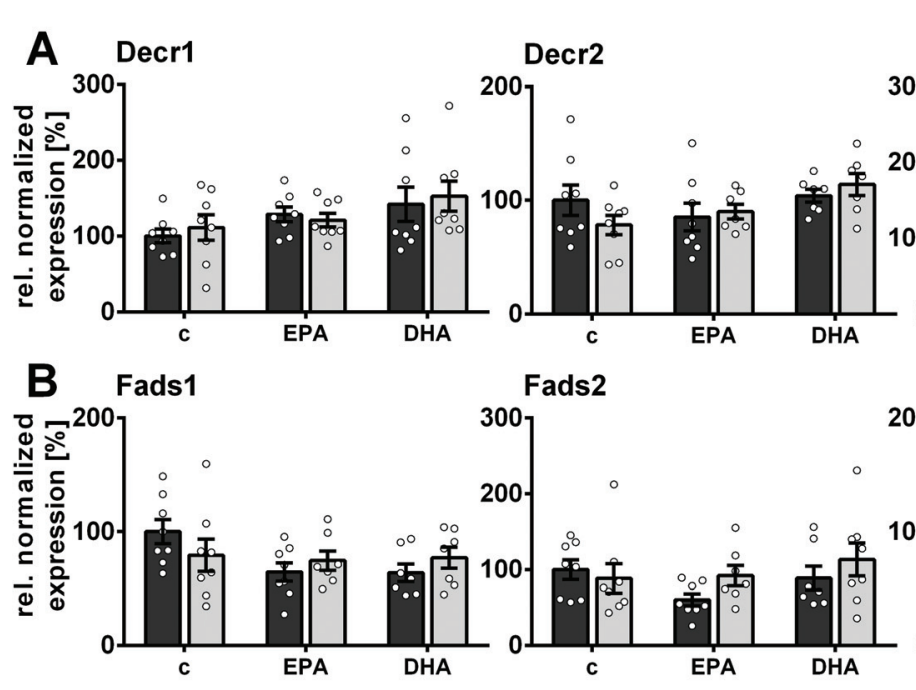

Fads2
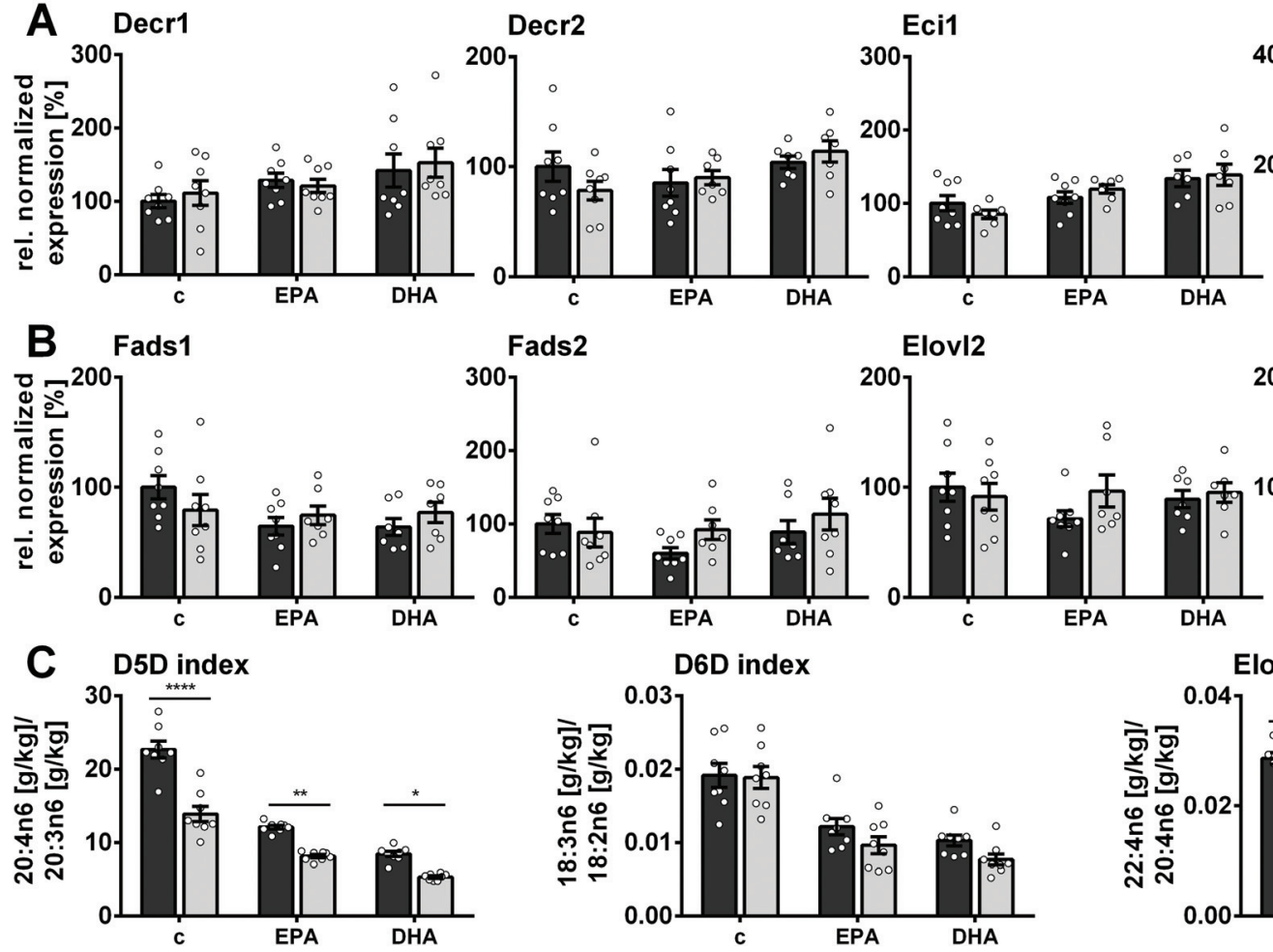

Eci2

n6-high $\square$ n6-low
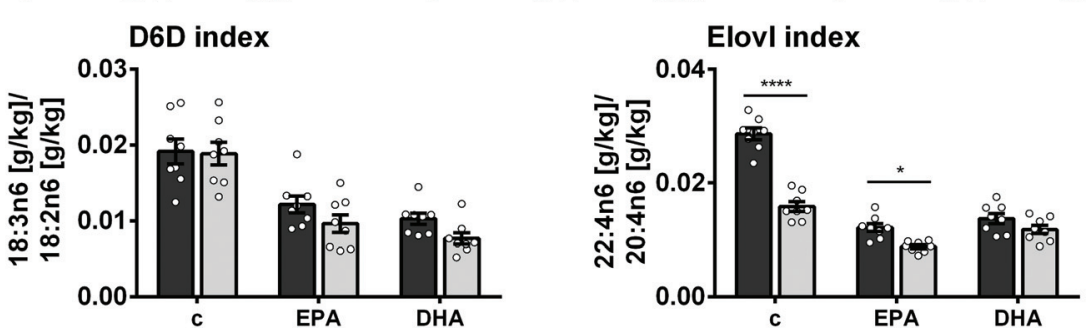

Fig. 3 Gene expression of enzymes and enzyme activity indices in mouse liver. Normalized gene expression relative to the c/n6-high group of enzymes involved in fatty acid $\beta$-oxidation, desaturation and elongation in liver tissue of NMRI mice after 28 days of feeding an n6-PUFA-rich diet (dark grey) or an n6-PUFA-low diet (light grey) without (c) or with n3-PUFA supplementation (EPA, DHA). Shown are mean \pm SEM ( $n=6-8)$ as well as individual values for (A) 2,4-dienoyl-CoA reductase 1 and 2 (Decr1, Decr2), cis- $\Delta^{3}$-enoyl-CoA isomerase 1 and 2 (Eci1, Eci2), (B) $\Delta 5-$ and $\Delta 6$-fatty acid desaturase (Fads1, Fads2), elongase 2 and 5 (Elovl2, Elovl5) and (C) fatty acid product/precursor ratio of C20:4n6/C20:3n6, C18:3n6/C18:2n6 and C22:4n6/C20:4n6 as estimates for enzyme activity of $\Delta 5$ - and $\Delta 6$-fatty acid desaturase (D5D, D6D) and elongase, respectively. Statistically significant differences $\left({ }^{*} p<0.05 ;{ }^{* *} p<0.01\right.$; $\left.{ }^{* \star} p<0.001 ;{ }^{* \star \star *} p<0.0001\right)$ were determined by two-way ANOVA with Tukey's post-test and are indicated for $n 6$-high vs. n6-low groups (results for comparisons of all groups are summarized in the ESI, Table S8 †). For relative gene expression data outliers were removed based on ROUT outlier test $(Q=1 \%)$. 


\subsection{Oxylipin concentrations in liver tissue}

In liver tissue, the trends of concentrations and the diet induced changes of ARA, EPA and DHA derived oxylipins were overall similar as their precursor PUFA, as shown for a set of representative oxylipins covering all branches of enzymatic oxylipin formation within the ARA cascade (Fig. 4; all quantified oxylipins are summarized in the ESI, Table S7†). Levels of ARA
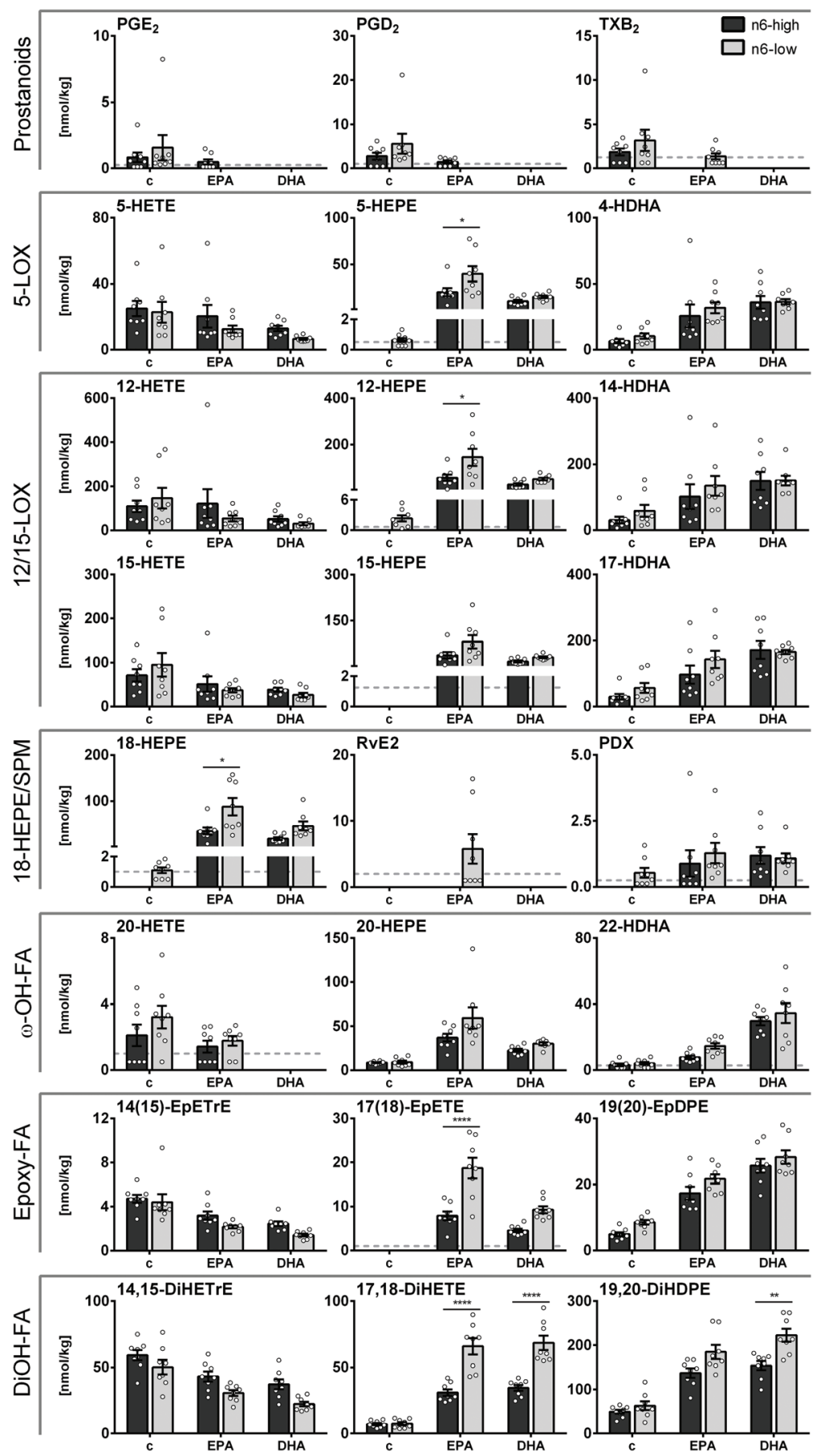

Fig. 4 Oxylipin concentrations in mouse liver tissue. Concentrations [nmol $\mathrm{kg}^{-1}$ ] of selected oxylipins derived from ARA, EPA and DHA including COX derived prostanoids, 5-, 12- and 15-LOX products, SPMs and SPM precursor, CYP derived epoxy- and $\omega$-hydroxy-FA as well as CYP/seH derived dihydroxy-FA in liver tissue of NMRI mice after 28 days of feeding an n6-PUFA-rich diet (dark grey) or an n6-PUFA-low diet (light grey) without (c) or with n3-PUFA supplementation (EPA, DHA). Shown are mean \pm SEM as well as individual values $(n=8)$. If $>50 \%$ of the samples within one group were $\angle$ LLOQ (lower limit of quantification), no mean was calculated and the LLOQ is indicated as dotted line. Statistically significant differences ${ }^{*} p$ $<0.05$; ${ }^{* *} p<0.01 ;{ }^{* * *} p<0.001$; ${ }^{* * *} p<0.0001$ ) were determined by two-way ANOVA with Tukey's post-test and are indicated for n6-high vs. n6low groups (results for comparisons of all groups are summarized in the ESI, Table S $8 \dagger$ ). 
derived oxylipins did generally not differ significantly between c/n6-high and c/n6-low groups (Fig. 4), which is consistent with ARA concentrations in liver tissue (Fig. 2 and ESI Table S4A-1). Prostanoids, i.e. $\mathrm{PGE}_{2}, \mathrm{PGD}_{2}$, $\mathrm{TXB}_{2}$, were very low in control groups and mostly undetectable in n3-PUFA-fed groups (Fig. 4). ARA derived 15-LOX product (15-HETE) was reduced upon DHA feeding combined with an n6-PUFA-low background diet. The 5-LOX and 12-LOX products 5-HETE and 12-HETE showed a consistent trend towards lower levels, which was not significant due to high inter-individual variation. CYP derived 14(15)-EpETrE and its SEH product 14,15DiHETrE were reduced by both EPA and DHA feeding, (Fig. 4, ESI Tables S7 and S8 $\dagger$ ).

EPA derived oxylipins were very low or $<$ LLOQ in both control groups (c/n6-high and c/n6-low) and were elevated by n3-PUFA supplementation. Particularly EPA feeding resulted in higher HEPE concentrations in liver tissue, which was more pronounced for the n6-low basal diet for 5-, 12- and 18-HEPE. In contrast to EPA tissue levels, EPA supplementation resulted in higher levels of EPA derived LOX products compared to DHA in n6-low groups. The effect of highest EPA derived oxylipin levels in the EPA/n6-low group could also be observed for SPM precursor 18-HEPE and consistently SPM RvE2 exceeded the LLOQ in $50 \%$ of the animals within this group (Fig. 4) and consistent MRM transitions support its identity (ESI Fig. S4†). EPA derived CYP/sEH products 17(18)-EpETE and 17,18DiHETE were stronger elevated in EPA/n6-low compared to EPA/n6-high groups.

Consistent with DHA liver concentrations, DHA derived LOX products (HDHAs) were not different between the n6-high and n6-low groups and increased upon both EPA and DHA feeding, though this was not significant for EPA/n6-high group or 14-HDHA. CYP products of DHA were similarly modulated upon n3-PUFA feeding and only the sEH product 19,20DiHDPE was higher in the DHA/n6-low compared to DHA/n6high group (Fig. 4).

\section{Discussion}

In the present study, we addressed the following questions: (i) does a replacement of dietary LA with OA lead to a reduction of tissue ARA concentrations, (ii) how are tissue levels of supplemented EPA and DHA affected by a low n6-PUFA background diet, (iii) are the changes attributable to hepatic expression of lipid-metabolizing enzymes and (iv) how does an n6-PUFA low background diet affect the modulation of the tissue free oxylipin profile.

\subsection{Low dietary LA and high OA has no effect on tissue ARA concentration}

The n3-PUFA status is associated with a lower risk for cardiovascular and inflammatory diseases. ${ }^{3,4}$ This might be in part linked to a reduced formation of pro-inflammatory n6-PUFA derived lipid mediators, such as 2-series prostaglandins or 4-series leukotrienes, due to competition of n3- and n6-PUFA for enzymatic conversion. Besides increasing n3-PUFA intake, lower levels of ARA and ARA derived lipid mediators might be achieved by decreasing the intake of n6-PUFA via the diet. Despite a strong reduction of LA concentrations in liver tissue of the c/n6-low group, tissue ARA concentrations were not affected. The D6D enzyme, which catalyzes the first step in LAto-ARA conversion, i.e. desaturation of LA to GLA, is discussed as the rate-limiting enzyme in this conversion. ${ }^{1}$ It seems likely that the LA content $(\sim 10 \%)$ in the n6-low dietary oil is sufficient for an effective supply with LA and further increase of LA does not enhance ARA production due to saturation of the D6D. Similarly, in a systematic review, no correlation was found between LA intake and ARA levels in humans on a Western diet, while intake of GLA (D6D product of LA) was found to increase ARA. ${ }^{36}$ Lower concentrations of ARA elongation/desaturation products, AdA and n6-DPA, in the c/n6-low group also indicate an intent of maintaining liver ARA levels by slower ARA conversion. Moreover, higher liver OA, which presumably accumulated in the neutral lipid fraction as shown for mouse and rat liver, ${ }^{37,38}$ might not be able to replace ARA from hepatic phospholipids. It should be noted that, while the liver tissue was not macroscopically different from the n6-high groups, the OA-rich diet led to an increase of total liver FA in NMRI mice. Total FA levels in the c/n6-low group were comparable to total fat in liver of C57BL/6J mice fed with a high fat diet $\left(60 \%\right.$ fat). ${ }^{39,40}$ However, increase in liver FA by OA feeding might be a mouse-specific effect and may not be transferable to humans.

\subsection{Elevation of blood and tissue EPA upon n3-PUFA feeding is more efficient when dietary LA is low}

Regarding n3-PUFA supplementation, an effective uptake and elevation of blood and tissue n3-PUFA levels as well as subsequent metabolization to bioactive lipid mediators might be influenced by the ratio of (competing) n6- and n3-PUFA in the diet. Supplementation of the n6-low, OA-rich diet with n3PUFA resulted in a trend towards lower total FA in liver tissue and levels of n3-PUFA were elevated at the expense of MUFA indicating an efficient uptake. In C57BL/6J mice on a high-fat/ high-sucrose diet, elevated OA content in total liver lipids was decreased by administration of EPA-EE, which also resulted in a reduction of the hepatic TG content. ${ }^{41}$

The more effective elevation of EPA with an n6-low background diet was evident in both the EPA- and DHA-fed groups. The levels of n3-DPA, which were also elevated by n3-PUFA feeding, however, without further elevation by the n6-low diet, indicate a slower enzymatic elongation of EPA to n3-DPA and thereby causing an accumulation of EPA. In human erythrocytes, increased EPA + DHA levels were observed when dietary LA was low and might be attributed to a more effective ALA-toSDA or C24:5n3-to-C24:6n3 conversion or competition for esterification into membrane phospholipids. ${ }^{23}$ An increased ALA-to-EPA conversion on an n6-low background may be excluded here, as ALA and eicosatetraenoic acid (ETA, C20:4n3) levels were overall low in liver tissue $(<0.05 \%$, ESI Fig. S1†). 
A similar increase of EPA levels regardless of supplementation with EPA or DHA was unexpected, even though the DHA diet contained a small amount of EPA. This effect was specifically observed in the liver. Elevation of tissue EPA when DHA is supplemented is in line with previous studies e.g. in rat liver phospholipids ${ }^{17}$ and in rat liver total fat dietary DHA led to a comparable increase of EPA levels as dietary EPA. ${ }^{42}$ DHA may be retro-converted to EPA in the mouse liver tissue as observed in human HepG2 cells. $^{43}$ Elevated mRNA levels of genes encoding for auxiliary enzymes for $\beta$-oxidation of PUFA, Eci1 and Eci2, in DHA/n6-low group compared to control group could indicate an increased $\beta$-oxidation of unsaturated FA and/ or retro-conversion in liver of DHA fed mice. Also, the elevation of EPA could result from a modulation of the ALA-EPA-DHA turn-over, e.g. a slower elongation, desaturation or $\beta$-oxidation of EPA in DHA supplemented groups ${ }^{44,45}$ and lower n3-DPA levels in DHA-fed $v s$. EPA-fed groups despite similar EPA concentrations could give a hint to a slower EPA elongation.

In contrast to EPA, DHA levels were not influenced by lowering the n6-PUFA content of the diet. Both DHA and EPA feeding resulted in an elevation of DHA for the two background diets. DHA could be formed from EPA either via a pathway involving elongation, desaturation and peroxisomal $\beta$-oxidation as reviewed by Sprecher $(2000)^{46}$ or a delta- 4 desaturation by D6D as shown for human cells. ${ }^{47}$ Although, it is believed that this conversion is relatively inefficient, it might occur with higher rates in rats and mice. ${ }^{27}$ Taken together, in the used model the n6-PUFA content of the diet had a clear influence on EPA levels, while DHA was unaffected.

\subsection{Hepatic elongase and desaturase mRNA levels are not significantly affected by a diet high in LA or OA}

The observed reduction of tissue and blood ARA levels upon n3-PUFA feeding is consistent with studies in mice and rats, in which both EPA + DHA are administered (2-3\% n3-PUFA oil and $4-10 \%$ fat in the diet). ${ }^{16,48,49}$ Interestingly, while there was little or no influence of the n6-PUFA background diet alone on ARA levels, the ARA-lowering effect of EPA and DHA supplementation was more pronounced in combination with reduced dietary LA. This might be a consequence of a competition between ARA and elevated EPA on the n6-low background or the combination of dietary n3-PUFA, low dietary LA and consequently high OA might affect the enzymatic conversion of LA-to-ARA (and ARA-to-n6-DPA). In order to determine if these effects are a result of substrate availability and/or enzyme saturation or if the diets influence the enzyme expression, we analyzed the gene expression (qPCR) of the involved elongases and desaturases in liver. These enzymes are required for the synthesis of C20 and C22 n3- and n6-PUFA from the essential FA LA and ALA in mammals and several steps are catalyzed by D5D (Fads1) and D6D (Fads2) as well as elongase 2 and 5 (Elovl2, Elovl5). ${ }^{50}$ Moreover, expression of these enzymes may be regulated by the dietary n3-PUFA intake. For example, a diet deprived in n3-PUFA resulted in upregulated desaturase (D5D, D6D) and elongase (Elovl2, Elovl5) activity and expression in rat liver. ${ }^{51}$ The product/pre- cursor ratio of GLA/LA or the D6D-index indicates a low but similarly efficient desaturation of both high and low LA levels, which decreases with n3-PUFA feeding. However, the D6Dindex showed no correlation with the Fads2 gene expression in the present study. It should be noted that enzyme activity estimates based on product/precursor FA ratios also depend on the PUFA intake. ${ }^{34}$ In rat liver, no modulation of hepatic elongase and desaturase gene expression by different dietary ALA levels was observed by Tu et al. (2010) and it was concluded that substrate availability and competition are more likely regulating n3-PUFA synthesis than different enzyme expression. ${ }^{52}$ In contrast, a replacement of dietary PUFA by MUFA increased Fads2 and Elovl2 significantly in rat liver, ${ }^{52}$ which we did not observe in mouse liver. Despite differences between FA metabolism in rats and mice, one possible explanation is that $10 \%$ PUFA (of total FA) in the c/n6-low diet in our study was high enough and therefore did not result in increased Fads2 expression.

The ARA/DGLA ratio or D5D-index was decreased by both n6-PUFA lowering and n3-PUFA elevation, resulting in an accumulation of DGLA rather than ARA. Similar to Fads2, no significant modulation of Fads1 mRNA levels was observed. The ratio of $\mathrm{C} 22: 4 \mathrm{n} 6 / \mathrm{C} 20: 4 \mathrm{n} 6$ which can be used as estimate for elongase activity ${ }^{34}$ is - like the D5D-index - reduced by both the n6-low diet and n3-PUFA supplementation indicating a slower elongation of ARA.

Taken together, we observed that a drastic shift from dietary (n6-) PUFA to (n9-) MUFA had no effect on transcription of desaturases and elongases involved in PUFA metabolism in liver tissue. This indicates that in the used experimental model, tissue FA composition is likely governed by substrate availability and competition.

\subsection{Modulation of tissue PUFA is reflected in the tissue oxylipin pattern}

Both n3- and n6-PUFA compete for the enzymatic oxidation and distinct substrate preferences or different potencies of n3and n6-PUFA derived lipid mediators have been described. For example, COX reacts slower with EPA, ${ }^{53}$ while CYP epoxygenases $^{54}$ and ALOX12/15 orthologs $^{55}$ are more efficient with EPA and DHA as substrate compared to ARA. Therefore, we investigated if the modulation of the tissue oxylipin profile was consistent with the FA pattern and if the low n6-PUFA background reduces the formation of predominantly pro-inflammatory lipid mediators derived from ARA. ${ }^{13}$ In general, oxylipins derived from ARA, EPA and DHA displayed similar trends as the respective precursor PUFA indicating that the total tissue FA composition governs oxylipin formation.

Levels of ARA derived COX metabolites in liver tissue were overall low and therefore it is not possible to conclude on a significant effect by the dietary intervention in these healthy, non-inflamed mice. Particularly, ARA derived lipid mediators of the CYP pathway were consistent with ARA tissue levels not influenced by the background diet alone, however, reduced by n3-PUFA feeding. In mouse liver, fish oil feeding resulted in a reduction of liver ARA derived oxylipins, ${ }^{48}$ and in rat liver ARA 
derived oxylipins were reduced with higher efficacy by DHA feeding compared to EPA feeding, ${ }^{17}$ which was not significant in our study.

Furthermore, some pathways indicate a preferred enzymatic oxidation of n3-PUFA over n6-PUFA. For example, despite tissue ARA levels (control groups) and DHA levels (after n3PUFA feeding) were in the same concentration range, clearly higher levels of DHA derived CYP product 19(20)-EpDPE was formed, which is consistent with a preference for the n3- over the n6-double bond by CYP epoxygenases as mechanistically described for human CYPs. ${ }^{49,56}$ Moreover, despite similar DHA and 19(20)-EpDPE concentrations in DHA/n6-high and DHA/ n6-low groups, higher levels of its SEH product may indicate a more efficient formation of DHA derived CYP products with an n6-low background diet. For 19(20)-EpDPE, which was the most abundant EpDPE in mouse liver, inhibitory effects on angiogenesis, tumor growth and metastasis, ${ }^{57}$ protection against LPS induced cytotoxicity ${ }^{58}$ and reduction of renal fibro$\operatorname{sis}^{59}$ were observed. In our study, a relatively low dose of n3PUFA led to an elevation of 19(20)-EpDPE tissue levels (2.8-3.5fold) indicating that both EPA and DHA feeding might exert protective effects via this bioactive lipid mediator.

In contrast to hepatic EPA levels, which were similar in EPA- and DHA-fed groups, increase in hepatic EPA derived oxylipin levels was more pronounced in EPA/n6-low fed groups. The divergence between tissue EPA and EPA derived oxylipins may result from their distribution in hepatic lipid pools. The phospholipid pool is believed to provide substrate FA for the formation of free oxylipins after release by phospholipases. ${ }^{6}$ However, as we analyzed total hepatic FA it is possible that elevated EPA in DHA-fed groups is directed to different lipid classes, e.g. hepatic TG and therefore not readily available for oxylipin formation. Moreover, oxylipins can be re-esterified to lipids or conjugated with glucuronic acid ${ }^{60}$ and free oxylipins represent only a small fraction of the total oxylipin profile. ${ }^{61}$ Other explanations could be an increased degradation/metabolization of EPA derived oxylipins in DHA-fed groups or a limited hepatic formation of EPA oxylipins when DHA is supplemented.

Highest levels of HEPEs and EpETEs were achieved by combining dietary EPA and low n6-PUFA. For example, 18-HEPE, a common pathway marker for E-series resolvin formation, is 80-fold elevated in the EPA/n6-low group compared to levels of the n6-low control group. For 18-HEPE itself an anti-inflammatory activity is described, such as the suppression of LPS-triggered TNF- $\alpha$ formation in murine macrophages ${ }^{62}$ or lowering of IL- 6 formation in murine cardiac fibroblasts stimulated with macrophage conditioned media. ${ }^{63}$ Moreover, EPA feeding especially in combination with low dietary n6-PUFA might be able to increase E-series resolvins, e.g. RvE2 that correlated with 18-HEPE formation and might exert anti-inflammatory properties such as reduction of neutrophil chemotactic velocity or enhancement of macrophage phagocytosis. ${ }^{64}$ Similarly, highest levels of CYP derived 17(18)-EpETE were observed in the EPA/n6-low group resulting in an $>18$-fold increase compared to the control groups, which were $<\operatorname{LLOQ}\left(<1 \mathrm{nmol} \mathrm{kg}^{-1}\right)$ in liver tissue. This oxylipin was also found to be an abundant
epoxy-FA in rat liver, heart and other tissues after n3-PUFA feeding and reduced spontaneous beating rate of neonatal cardiomyocytes more effectively than EPA regarding delay time and concentration. ${ }^{49}$ Our results indicate that elevation of 17 (18)-EpETE, which is investigated for its anti-arrhythmic effects, ${ }^{65}$ can be increased by combining EPA supplementation with low n6-PUFA intake. Interestingly, while 17(18)-EpETE levels were lower in the DHA/n6-low group, its sEH product 17,18-DiHETE was similarly elevated. Thus, also DHA feeding may efficiently raise 17(18)-EpETE, which however, was more rapidly hydrolyzed in mouse liver.

In summary, both the n3-PUFA feeding and the n6-PUFA background diet had a similar effect on the tissue oxylipin pattern compared to their precursor PUFA, except bioactive EPA derived lipid mediators, which were clearly elevated most efficiently by EPA/n6-low feeding.

\subsection{Combining low dietary LA and n3-PUFA supplementation efficiently lowers \%n6 in HUFA and increases blood cell \%EPA + DHA}

The n3-PUFA status or more precisely the n3-index (\%EPA + DHA in red blood cells) has been associated with cardioprotection. ${ }^{66}$ The \%n6 in HUFA was proposed as another biomarker for health risk assessment taking into account not only the n3PUFA status but also the n3/n6-PUFA balance (as summarized in ref. 67). In the present study, we observed an elevation of \% EPA + DHA and \%n3 in HUFA as well as a decrease of \%n6 in HUFA when supplementing n3-PUFA, which was more pronounced for n6-low groups (except \%EPA + DHA in liver).

$\% \mathrm{EPA}+$ DHA in blood cells of the control groups was $<4 \%$ regardless of the background diet, which - translated to humans - has been associated with low cardioprotection, i.e. with higher risk for primary cardiac arrest, sudden cardiac death or ischemic heart disease, while an n3-index of $\geq 8 \%$ is associated with cardioprotection. ${ }^{66}$ Supplementation with EPA or DHA resulted in an elevation of \%EPA + DHA in blood cells to $>8 \%$ and $>11 \%$ in n6-high and n6-low groups, respectively. Hence, when dietary n6-PUFA are reduced even a low supplementation with n3-PUFA $(<0.6 \%$ EPA or DHA) could be sufficient to increase $\% \mathrm{EPA}+$ DHA to levels $\geq 8 \%$.

The \%n6 in HUFA for North Americans ranges from $75-80 \%$ and a reduction towards $60 \%$ may have a positive effect on human health as summarized by Lands et al. (2018). ${ }^{35}$ In mice receiving either of the control diets the \%n6 in HUFA ranged between 77-91\% (tissue and blood) and could be reduced towards $60 \%$ for mice on the n6-high diet (50-63\%) and towards $40 \%$ for mice on the n6-low diet (33-49\%). From this it can be concluded that despite little changes of ARA levels when dietary LA is reduced, a combination with long-chain n3-PUFA is more effective in modifying biomarkers related to the n3-PUFA status and associated with beneficial health effects.

In conclusion, in a murine model, a shift in dietary FA from mainly n6-PUFA (LA) to MUFA (OA) affected the overall FA composition of liver tissue and blood. No effect on ARA and n3-PUFA concentrations or mRNA levels of hepatic elon- 
gases/desaturases was observed. Supplementation with $\sim 0.6 \%$ n3-PUFA (either EPA or DHA) lowered blood and tissue levels of ARA and its elongation product to a greater extend when dietary LA was low. Particularly EPA and EPA oxylipins but not DHA and DHA oxylipins were affected by the background diet and their increase was 2-3-fold more pronounced. This resulted in higher \%EPA + DHA in blood cells and lower \%n6 in HUFA in blood and liver for the n6-low background diet. Thus, lower doses of long-chain n3-PUFA supplementation especially for EPA - are equally effective when the n6-PUFA background is low.

\section{Limitations}

Results obtained from experimental animal models cannot simply be extrapolated to humans, which also limits the conclusions drawn from the present study. Mice have a different FA and oxylipin metabolism than humans, for example regarding the efficiency of ALA elongation or enzyme product specificity (e.g. ALOX15). Therefore, obtained FA and oxylipin patterns cannot reflect tissue levels in humans. In order to account for that, the dietary FA composition used in this study contained a very low concentration of ALA and thus is compared to human nutrition artificial. Particularly the unsupplemented control groups received consequently a diet with an extremely high n6/n3-PUFA ratio, which may have induced an n3-PUFA deficiency in these groups. It should be noted that there are differences in lipid, FA and oxylipin metabolism in males and females and the results described in the present study may not be (completely) transferable to male mice.

\section{Abbreviations}

$\begin{array}{ll}\text { AdA } & \text { Adrenic acid, C22:4n6 } \\ \text { ALA } & \text { Alpha-linolenic acid, C18:3n3 } \\ \text { ARA } & \text { Arachidonic acid, C20:4n6 } \\ \text { COX } & \text { Cyclooxygenase } \\ \text { CYP } & \text { Cytochrome P450 monooxygenase } \\ \text { D5D } & \Delta 5 \text {-Desaturase } \\ \text { D6D } & \Delta 6 \text {-Desaturase } \\ \text { DGLA } & \text { Dihomo-gamma linolenic acid, C20:3n6 } \\ \text { DHA } & \text { Docosahexaenoic acid, C22:6n3 } \\ \text { DiOH-FA } & \text { Dihydroxy fatty acid(s) } \\ \text { DiHDHA } & \text { Dihydroxy docosahexaenoic acid } \\ \text { DiHDPE } & \text { Dihydroxy docosapentaenoic acid } \\ \text { DiHEPE } & \text { Dihydroxy eicosapentaenoic acid } \\ \text { DiHETE } & \text { Dihydroxy eicosatetraenoic acid } \\ \text { DiHETrE } & \text { Dihydroxy eicosatrienoic acid } \\ \text { DiHODE } & \text { Dihydroxy octadecadienoic acid } \\ \text { DiHOME } & \text { Dihydroxy octadecenoic acid } \\ \text { DPA } & \text { Docosapentaenoic acid, C22:5n3/C22:5n6 } \\ \text { FA } & \text { Fatty acid(s) } \\ \text { GC-FID } & \text { Gas chromatography with flame ionization } \\ & \text { detection }\end{array}$

GLA Gamma-linolenic acid, C18:3n6

EPA Eicosapentaenoic acid, C20:5n3

Ep-FA Epoxy fatty acid(s)

EpDPE Epoxy docosapentaenoic acid

EpETE Epoxy eicosatetraenoic acid

EpETrE Epoxy eicosatrienoic acid

EpODE Epoxy octadecadienoic acid

EPOME Epoxy octadecenoic acid

ETA Eicosatetraenoic acid, C20:4n3

HDHA Hydroxy docosahexaenoic acid

HEPE Hydroxy eicosapentaenoic acid

HETE Hydroxy eicosatetraenoic acid

HODE Hydroxy octadecadienoic acid

HOTrE Hydroxy octadecatrienoic acid

HUFA Highly unsaturated fatty acid(s)

LA Linoleic acid, C18:2n6

LC-MS/MS Liquid chromatography tandem mass spectrometry

LOX Lipoxygenase

MA Mead acid, C20:3n9

MUFA Monounsaturated fatty acid(s)

n3/n6/n9 Omega-3/6/9

OA Oleic acid, C18:1n9

OH-FA Hydroxy fatty acid(s)

PG Prostaglandin(s)

PUFA Polyunsaturated fatty acid(s)

SFA Saturated fatty acid(s)

SPM Specialized pro-resolving lipid mediator

TX Thromboxane(s)

\section{Conflicts of interest}

The authors declare that they have no conflicts of interest with the contents of this article.

\section{Acknowledgements}

This work was in part supported by a grant of the Deutsche Forschungsgemeinschaft (DFG) to NHS (Sche 1801). We would like to thank Annika I. Ostermann for helping to prepare the feeding oils.

\section{References}

1 J. Y. Zhang, K. S. D. Kothapalli and J. T. Brenna, Desaturase and elongase-limiting endogenous long-chain polyunsaturated fatty acid biosynthesis, Curr. Opin. Clin. Nutr., 2016, 19, 103-110.

2 P. C. Calder, Mechanisms of action of (n-3) fatty acids, J. Nutr., 2012, 142, 592S-599S.

3 D. Mozaffarian and J. H. Wu, Omega-3 fatty acids and cardiovascular disease: effects on risk factors, molecular 
pathways, and clinical events, J. Am. Coll. Cardiol., 2011, 58, 2047-2067.

4 P. C. Calder, Marine omega-3 fatty acids and inflammatory processes: Effects, mechanisms and clinical relevance, Biochim. Biophys. Acta, 2015, 1851, 469-484.

5 G. C. Burdge and P. C. Calder, Dietary alpha-linolenic acid and health-related outcomes: a metabolic perspective, Nutr. Res. Rev., 2006, 19, 26-52.

6 M. W. Buczynski, D. S. Dumlao and E. A. Dennis, An integrated omics analysis of eicosanoid biology (vol 50, pg 1015, 2009), J. Lipid Res., 2009, 50, 1505-1505.

7 C. Gladine, A. I. Ostermann, J. W. Newman and N. H. Schebb, MS-based targeted metabolomics of eicosanoids and other oxylipins: Analytical and inter-individual variabilities, Free Radicals Biol. Med., 2019, 144, 72-89.

8 J. D. Morrow, J. A. Awad, H. J. Boss, I. A. Blair and L. J. Roberts, 2nd, Non-cyclooxygenase-derived prostanoids (F2-isoprostanes) are formed in situ on phospholipids, Proc. Natl. Acad. Sci. U. S. A., 1992, 89, 10721-10725.

9 K. M. Rund, D. Heylmann, N. Seiwert, S. Wecklein, C. Oger, J. M. Galano, T. Durand, R. Chen, F. Gueler, J. Fahrer, J. Bornhorst and N. H. Schebb, Formation of trans-epoxy fatty acids correlates with formation of isoprostanes and could serve as biomarker of oxidative stress, Prostaglandins Other Lipid Mediators, 2019, 144, 106334.

10 A. P. Simopoulos, The importance of the ratio of omega-6/ omega-3 essential fatty acids, Biomed. Pharmacother., 2002, 56, 365-379.

11 A. C. Beynen and M. B. Katan, Why do polyunsaturated fatty acids lower serum cholesterol?, Am. J. Clin. Nutr., 1985, 42, 560-563.

12 B. Lands, Historical perspectives on the impact of n-3 and n-6 nutrients on health, Prog. Lipid Res., 2014, 55, 17-29.

13 B. Lands, Omega-3 PUFAs Lower the Propensity for Arachidonic Acid Cascade Overreactions, BioMed Res. Int., 2015, 2015, 285135.

14 L. M. Browning, C. G. Walker, A. P. Mander, A. L. West, J. Madden, J. M. Gambell, S. Young, L. Wang, S. A. Jebb and P. C. Calder, Incorporation of eicosapentaenoic and docosahexaenoic acids into lipid pools when given as supplements providing doses equivalent to typical intakes of oily fish, Am. J. Clin. Nutr., 2012, 96, 748-758.

15 A. I. Ostermann, A. L. West, K. Schoenfeld, L. M. Browning, C. G. Walker, S. A. Jebb, P. C. Calder and N. H. Schebb, Plasma oxylipins respond in a linear dose-response manner with increased intake of EPA and DHA: results from a randomized controlled trial in healthy humans, Am. J. Clin. Nutr., 2019, 109, 1251-1263.

16 A. I. Ostermann, P. Waindok, M. J. Schmidt, C. Y. Chiu, C. Smyl, N. Rohwer, K. H. Weylandt and N. H. Schebb, Modulation of the endogenous omega-3 fatty acid and oxylipin profile in vivo-A comparison of the fat- 1 transgenic mouse with $\mathrm{C} 57 \mathrm{BL} / 6$ wildtype mice on an omega-3 fatty acid enriched diet, PLoS One, 2017, 12, e0184470.

17 S. Leng, T. Winter and H. M. Aukema, Dietary ALA, EPA and DHA have distinct effects on oxylipin profiles in female and male rat kidney, liver and serum, J. Nutr. Biochem., 2018, 57, 228-237.

18 T. Terano, J. A. Salmon and S. Moncada, Biosynthesis and biological activity of leukotriene B5, Prostaglandins, 1984, 27, 217-232.

19 C. N. Serhan and N. A. Petasis, Resolvins and protectins in inflammation resolution, Chem. Rev., 2011, 111, 59225943.

20 K. E. Wood, E. Mantzioris, R. A. Gibson, C. E. Ramsden and B. S. Muhlhausler, The effect of modifying dietary LA and ALA intakes on omega-3 long chain polyunsaturated fatty acid (n-3 LCPUFA) status in human adults: A systematic review and commentary, Prostaglandins, Leukotrienes Essent. Fatty Acids, 2015, 95, 47-55.

21 J. T. Brenna, N. Salem, A. J. Sinclair, S. C. Cunnane and International Society for the Study of Fatty Acids and Lipids (ISSFAL), alpha-Linolenic acid supplementation and conversion to n-3 long-chain polyunsaturated fatty acids in humans, Prostaglandins, Leukotrienes Essent. Fatty Acids, 2009, 80, 85-91.

22 A. Y. Taha, Y. Cheon, K. F. Faurot, B. Macintosh, S. F. Majchrzak-Hong, J. D. Mann, J. R. Hibbeln, A. Ringel and C. E. Ramsden, Dietary omega- 6 fatty acid lowering increases bioavailability of omega-3 polyunsaturated fatty acids in human plasma lipid pools, Prostaglandins, Leukotrienes Essent. Fatty Acids, 2014, 90, 151-157.

23 B. A. MacIntosh, C. E. Ramsden, K. R. Faurot, D. Zamora, M. Mangan, J. R. Hibbeln and J. D. Mann, Low-n-6 and low-n-6 plus high-n-3 diets for use in clinical research, Br. J. Nutr., 2013, 110, 559-568.

24 C. B. Dias, L. G. Wood and M. L. Garg, Effects of dietary saturated and n-6 polyunsaturated fatty acids on the incorporation of long-chain n-3 polyunsaturated fatty acids into blood lipids, Eur. J. Clin. Nutr., 2016, 70, 812-818.

25 J. Giacometti, C. Milin, M. Tota, M. Cuk and B. RadosevicStasic, Incorporation of fatty acids into tissue phospholipids in mice fed diets rich in n-9 and n-6 fatty acids, Croat. Chem. Acta, 2005, 78, 397-404.

26 J. Hwang, H. S. Jun and E. Shim, Rates of Change in Tissue Fatty Acid Composition When Dietary Soybean Oil Is Switched to Olive Oil, J. Health Sci., 2010, 56, 275286.

27 B. M. Anderson and D. W. L. Ma, Are all n-3 polyunsaturated fatty acids created equal?, Lipids Health Dis., 2009, 8, 33.

28 B. J. Prendergast, K. G. Onishi and I. Zucker, Female mice liberated for inclusion in neuroscience and biomedical research, Neurosci. Biobehav. Rev., 2014, 40, 1-5.

29 A. I. Ostermann, M. Muller, I. Willenberg and N. H. Schebb, Determining the fatty acid composition in plasma and tissues as fatty acid methyl esters using gas chromatography - a comparison of different derivatization and extraction procedures, Prostaglandins, Leukotrienes Essent. Fatty Acids, 2014, 91, 235-241.

30 A. I. Ostermann, M. Reutzel, N. Hartung, N. Franke, L. Kutzner, K. Schoenfeld, K. H. Weylandt, G. P. Eckert and N. H. Schebb, A diet rich in omega-3 fatty acids enhances 
expression of soluble epoxide hydrolase in murine brain, Prostaglandins Other Lipid Mediators, 2017, 133, 79-87.

31 K. M. Rund, A. I. Ostermann, L. Kutzner, J. M. Galano, C. Oger, C. Vigor, S. Wecklein, N. Seiwert, T. Durand and N. H. Schebb, Development of an LC-ESI(-)-MS/MS method for the simultaneous quantification of 35 isoprostanes and isofurans derived from the major n3-and n6-PUFAs, Anal. Chim. Acta, 2018, 1037, 63-74.

32 L. Kutzner, K. M. Rund, A. I. Ostermann, N. M. Hartung, J. M. Galano, L. Balas, T. Durand, M. S. Balzer, S. David and N. H. Schebb, Development of an Optimized LC-MS Method for the Detection of Specialized Pro-Resolving Mediators in Biological Samples, Front. Pharmacol., 2019, 10, 169.

33 M. Lankinen, M. Uusitupa and U. Schwab, Genes and Dietary Fatty Acids in Regulation of Fatty Acid Composition of Plasma and Erythrocyte Membranes, Nutrients, 2018, 10, 1785.

34 H. Cormier, I. Rudkowska, S. Lemieux, P. Couture, P. Julien and M. C. Vohl, Effects of FADS and ELOVL polymorphisms on indexes of desaturase and elongase activities: results from a pre-post fish oil supplementation, Genes Nutr., 2014, 9, 437.

35 B. Lands, D. Bibus and K. D. Stark, Dynamic interactions of n-3 and n-6 fatty acid nutrients, Prostaglandins, Leukotrienes Essent. Fatty Acids, 2018, 136, 15-21.

36 B. S. Rett and J. Whelan, Increasing dietary linoleic acid does not increase tissue arachidonic acid content in adults consuming Western-type diets: a systematic review, Nutr. Metab., 2011, 8, 36.

37 M. J. Picklo, J. Idso, D. R. Seeger, H. M. Aukema and E. J. Murphy, Comparative effects of high oleic acid vs high mixed saturated fatty acid obesogenic diets upon PUFA metabolism in mice, Prostaglandins, Leukotrienes Essent. Fatty Acids, 2017, 119, 25-37.

38 A. Artmann, G. Petersen, L. I. Hellgren, J. Boberg, C. Skonberg, C. Nellemann, S. H. Hansen and H. S. Hansen, Influence of dietary fatty acids on endocannabinoid and $\mathrm{N}$-acylethanolamine levels in rat brain, liver and small intestine, Biochim. Biophys. Acta, Mol. Cell Biol. Lipids, 2008, 1781, 200-212.

39 R. Valenzuela, C. Barrera, A. Espinosa, P. Llanos, P. Orellana and L. A. Videla, Reduction in the desaturation capacity of the liver in mice subjected to high fat diet: Relation to LCPUFA depletion in liver and extrahepatic tissues, Prostaglandins, Leukotrienes Essent. Fatty Acids, 2015, 98, 7-14.

40 M. A. Rincon-Cervera, R. Valenzuela, M. C. HernandezRodas, M. Marambio, A. Espinosa, S. Mayer, N. Romero, C. Barrera, A. Valenzuela and L. A. Videla, Supplementation with antioxidant-rich extra virgin olive oil prevents hepatic oxidative stress and reduction of desaturation capacity in mice fed a high-fat diet: Effects on fatty acid composition in liver and extrahepatic tissues, Nutrition, 2016, 32, 12541267.
41 S. Kajikawa, T. Harada, A. Kawashima, K. Imada and K. Mizuguchi, Highly purified eicosapentaenoic acid prevents the progression of hepatic steatosis by repressing monounsaturated fatty acid synthesis in high-fat/highsucrose diet-fed mice, Prostaglandins, Leukotrienes Essent. Fatty Acids, 2009, 80, 229-238.

42 H. Poudyal, S. K. Panchal, L. C. Ward and L. Brown, Effects of ALA, EPA and DHA in high-carbohydrate, high-fat dietinduced metabolic syndrome in rats, J. Nutr. Biochem., 2013, 24, 1041-1052.

43 H. G. Park, P. Lawrence, M. G. Engel, K. Kothapalli and J. T. Brenna, Metabolic fate of docosahexaenoic acid (DHA; 22:6n-3) in human cells: direct retroconversion of DHA to eicosapentaenoic acid (20:5n-3) dominates over elongation to tetracosahexaenoic acid (24:6n-3), FEBS Lett., 2016, 590, 3188-3194.

44 A. H. Metherel, R. Chouinard-Watkins, M. O. Trepanier, R. J. S. Lacombe and R. P. Bazinet, Retroconversion is a minor contributor to increases in eicosapentaenoic acid following docosahexaenoic acid feeding as determined by compound specific isotope analysis in rat liver, Nutr. Metab., 2017, 14, 75.

45 A. H. Metherel, M. Irfan, S. L. Klingel, D. M. Mutch and R. P. Bazinet, Compound-specific isotope analysis reveals no retroconversion of DHA to EPA but substantial conversion of EPA to DHA following supplementation: a randomized control trial, Am. J. Clin. Nutr., 2019, 110, 823831.

46 H. Sprecher, Metabolism of highly unsaturated n-3 and n-6 fatty acids, Biochim. Biophys. Acta, Mol. Cell Biol. Lipids, 2000, 1486, 219-231.

47 H. G. Park, W. J. Park, K. S. D. Kothapalli and J. T. Brenna, The fatty acid desaturase 2 (FADS2) gene product catalyzes Delta 4 desaturation to yield n-3 docosahexaenoic acid and n-6 docosapentaenoic acid in human cells, FASEB J., 2015, 29, 3911-3919.

48 M. G. J. Balvers, K. C. M. Verhoeckx, S. Bijlsma, C. M. Rubingh, J. Meijerink, H. M. Wortelboer and R. F. Witkamp, Fish oil and inflammatory status alter the n-3 to n- 6 balance of the endocannabinoid and oxylipin metabolomes in mouse plasma and tissues, Metabolomics, 2012, 8, 1130-1147.

49 C. Arnold, M. Markovic, K. Blossey, G. Wallukat, R. Fischer, R. Dechend, A. Konkel, C. von Schacky, F. C. Luft, D. N. Muller, M. Rothe and W. H. Schunck, Arachidonic Acid-metabolizing Cytochrome P450 Enzymes Are Targets of omega-3 Fatty Acids, J. Biol. Chem., 2010, 285, 3272032733.

50 H. Guillou, D. Zadravec, P. G. Martin and A. Jacobsson, The key roles of elongases and desaturases in mammalian fatty acid metabolism: Insights from transgenic mice, Prog. Lipid Res., 2010, 49, 186-199.

51 M. Igarashi, K. Ma, L. Chang, J. M. Bell and S. I. Rapoport, Dietary n-3 PUFA deprivation for 15 weeks upregulates elongase and desaturase expression in rat liver but not brain, J. Lipid Res., 2007, 48, 2463-2470. 
52 W. C. Tu, R. J. Cook-Johnson, M. J. James, B. S. Muhlhausler and R. A. Gibson, Omega-3 long chain fatty acid synthesis is regulated more by substrate levels than gene expression, Prostaglandins, Leukotrienes Essent. Fatty Acids, 2010, 83, 61-68.

53 M. Wada, C. J. DeLong, Y. H. Hong, C. J. Rieke, I. Song, R. S. Sidhu, C. Yuan, M. Warnock, A. H. Schmaier, C. Yokoyama, E. M. Smyth, S. J. Wilson, G. A. FitzGerald, M. Garavito, D. X. Sui, J. W. Regan and W. L. Smith, Enzymes and receptors of prostaglandin pathways with arachidonic acid-derived versus eicosapentaenoic acid-derived substrates and products, J. Biol. Chem., 2007, 282, 2225422266.

54 R. Fischer, A. Konkel, H. Mehling, K. Blossey, A. Gapelyuk, N. Wessel, C. von Schacky, R. Dechend, D. N. Muller, M. Rothe, F. C. Luft, K. Weylandt and W. H. Schunck, Dietary omega-3 fatty acids modulate the eicosanoid profile in man primarily via the CYP-epoxygenase pathway, J. Lipid Res., 2014, 55, 1150-1164.

55 L. Kutzner, K. Goloshchapova, D. Heydeck, S. Stehling, H. Kuhn and N. H. Schebb, Mammalian ALOX15 orthologs exhibit pronounced dual positional specificity with docosahexaenoic acid, Biochim. Biophys. Acta, Mol. Cell Biol. Lipids, 2017, 1862, 666-675.

56 J. P. Schuchardt, S. Schmidt, G. Kressel, I. Willenberg, B. D. Hammock, A. Hahn and N. H. Schebb, Modulation of blood oxylipin levels by long-chain omega-3 fatty acid supplementation in hyper- and normolipidemic men, Prostaglandins, Leukotrienes Essent. Fatty Acids, 2014, 90, 27-37.

57 G. D. Zhang, D. Panigrahy, L. M. Mahakian, J. Yang, J. Y. Liu, K. S. S. Lee, H. I. Wettersten, A. Ulu, X. W. Hu, S. Tam, S. H. Hwang, E. S. Ingham, M. W. Kieran, R. H. Weiss, K. W. Ferrara and B. D. Hammock, Epoxy metabolites of docosahexaenoic acid (DHA) inhibit angiogenesis, tumor growth, and metastasis, Proc. Natl. Acad. Sci. U. S. A., 2013, 110, 6530-6535.

58 V. Samokhvalov, K. L. Jamieson, J. Vriend, S. Quan and J. M. Seubert, CYP epoxygenase metabolites of docosahexaenoic acid protect HL-1 cardiac cells against LPS-induced cytotoxicity through SIRT1, Cell Death Discovery, 2015, 1, 15054.
59 A. Sharma, M. A. H. Khan, S. P. Levick, K. S. S. Lee, B. D. Hammock and J. D. Imig, Novel Omega-3 Fatty Acid Epoxygenase Metabolite Reduces Kidney Fibrosis, Int. J. Mol. Sci., 2016, 17, 751.

60 D. Turgeon, S. Chouinard, P. Belanger, S. Picard, J. F. Labbe, P. Borgeat and A. Belanger, Glucuronidation of arachidonic and linoleic acid metabolites by human UDP-glucuronosyltransferases, J. Lipid Res., 2003, 44, 11821191.

61 A. I. Ostermann, E. Koch, K. M. Rund, L. Kutzner, M. Mainka and N. H. Schebb, Targeting esterified oxylipins by LC-MS Effect of sample preparation on oxylipin pattern, Prostaglandins Other Lipid Mediators, 2020, 146, 106384.

62 K. H. Weylandt, L. F. Krause, B. Gomolka, C. Y. Chiu, S. Bilal, A. Nadolny, S. F. Waechter, A. Fischer, M. Rothe and J. X. Kang, Suppressed liver tumorigenesis in fat1 mice with elevated omega- 3 fatty acids is associated with increased omega-3 derived lipid mediators and reduced TNF-alpha, Carcinogenesis, 2011, 32, 897-903.

63 J. Endo, M. Sano, Y. Isobe, K. Fukuda, J. X. Kang, H. Arai and M. Arita, 18-HEPE, an n-3 fatty acid metabolite released by macrophages, prevents pressure overloadinduced maladaptive cardiac remodeling, J. Exp. Med., 2014, 211, 1673-1687.

64 S. F. Oh, M. Dona, G. Fredman, S. Krishnamoorthy, D. Irimia and C. N. Serhan, Resolvin E2 Formation and Impact in Inflammation Resolution, J. Immunol., 2012, 188, 4527-4534.

65 A. M. Adebesin, T. Wesser, J. Vijaykumar, A. Konkel, M. P. Paudyal, J. Lossie, C. Zhu, C. Westphal, N. Puli, R. Fischer, W. H. Schunck and J. R. Falck, Development of Robust 17(R),18(S)-Epoxyeicosatetraenoic Acid (17,18-EEQ) Analogues as Potential Clinical Antiarrhythmic Agents, J. Med. Chem., 2019, 62, 10124-10143.

66 W. S. Harris and C. von Schacky, The Omega-3 Index: a new risk factor for death from coronary heart disease?, Prev. Med., 2004, 39, 212-220.

67 D. Bibus and B. Lands, Balancing proportions of competing omega-3 and omega-6 highly unsaturated fatty acids (HUFA) in tissue lipids, Prostaglandins, Leukotrienes Essent. Fatty Acids, 2015, 99, 19-23. 